\title{
EL MODELO DE POLICÍA COMUNITARIA: EL CASO CHILENO
}

\author{
THE MODEL OF COMMUNITY POLICING: THE CHILEAN CASE
}

Fecha de Recepción: 05/03/2011

Fecha de Aceptación: 10/03/2011

Cynthia Labra Díaz'

\section{Resumen:}

Se reflexiona sobre el policiamiento comunitario en el caso del plan cuadrante de Carabineros de Chile, analizando las bases, los principios y las características del modelo de policía de proximidad y su aplicación en el referido plan. Afirmo que el plan cuadrante posee solo algunos elementos del modelo.

Palabras clave: Policía comunitaria, criminalidad, estructura policial, actividades policiales en equipo.

\begin{abstract}
:
It think over about the community policing in the case of Carabineros de Chile's quadrant plan, analyzing the foundations, principles and characteristics of the proximity policing model and its application in this plan. I affim that the quadrant plan has only some elements of the model.
\end{abstract}

Key words: Community policing, criminality, police structure, team policing

\footnotetext{
Abogada y Licenciada en Ciencias Jurídicas, Universidad Católica de Temuco. Máster en Criminología y Ejecución Penal, Universidad Autónoma de Barcelona, España. Correo: cynthialabra@yahoo.es
} 


\section{Aproximación al modelo de policía comunitaria}

Las instituciones policiacas latinoamericanas son el corolario de una combinación de varios fenómenos. Se destaca la compleja relación existente entre la policía y el poder público, que en períodos prolongados de la historia ha padecido de características no democráticas. La policía se coloca al servicio del sistema político de turno; lo que implica una delgada línea entre el respeto al Estado de Derecho y el actuar policial. Además, las policías latinoamericanas aun son tributarias de un modelo de organización centralizado y de tipo militar, como sucede, por ejemplo, con las policías de Brasil, Carabineros (Chile) y con la Policía Nacional del Perú. Este tipo de organización facilita la mantención de la disciplina interna, pero requiere ajustes si se desea avanzar hacia modelos de administración más flexibles y que incentivan la cooperación con la comunidad ${ }^{2}$. Una policía militar por su formación institucional no es cercana a la comunidad, porque sus funciones están orientadas por el modelo de vigilancia represivo del cual la ciudadanía no forma parte. Estas características impelen a los cuerpos policiales a reorientar la institucionalidad y sus prácticas en aras de la percepción de la ciudadanía. El modelo de policiamiento comunitario es, entonces, una vía posible para lograr ese objetivo.

Este trabajo se aproxima al modelo de policía comunitaria, analiza las bases teóricas del modelo y reflexiona acerca de la incorporación de los principios en las actuaciones de la policía de Carabineros de Chile. Particularmente, en el plan cuadrante. El modelo de policía comunitaria o de proximidad es una oportunidad para el cambio en el actuar policial. El policiamiento comunitario es un modelo que resulta atractivo, porque es una herramienta de gestión útil, para mejorar el grado de aceptación de las instituciones policiales y de sus prácticas. Sí comprende, bajo la óptica de este modelo, que las necesidades particulares de una comunidad no pueden ser satisfechas a través de fórmulas centralizadas y militarizadas. Porque el fundamento del modelo de policía comunitario es mejorar la calidad de vida de las personas de acuerdo a una intervención acotada a sus necesidades reales, es decir, el policiamiento comunitario es un trabajo policial personalizado, en contacto directo con las personas "porque el modelo no está definido como una lista de actividades que entren en un marco definido, sino más bien por una estrategia de adaptación que responde a las exigencias de un entorno que esta en permanente evolución y dinamismo3."

Existe acuerdo, en el hecho que este modelo busca elevar la calidad de vida de la comunidad, mediante un conjunto de estrategias y técnicas, que se sustentan en tres ejes: conocer los intereses y necesidades locales, que a veces no coinciden con

Frühling, Hugo, Policía Comunitaria y Reforma Policial en América Latina. ¿Cuál es el impacto? Instituto de Estudios Públicos, Universidad de Chile, Santiago, 2003.

3 Skogan, Wesley, Réformer la Police et la sécurité. Les nouvelles tendences en Europe et États-Unis. Odile Jacob, 2004. 
las que la policía posee; proporcionar una oportunidad para educar a la ciudadanía acerca de los comportamientos que ayudan a prevenir la delincuencia y abrir un espacio para que los ciudadanos expresen directamente sus quejas ${ }^{4}$.

\section{Bases del modelo de policía comunitaria}

Para entender cómo aparece el modelo de policía comunitaria, hay que situarse dentro del contexto histórico en el que nace la policía. La Europa de los siglos XVIII y comienzo del XIX, en la cual surge la figura del denominado padre de la policía Londindense, Sir Robert Peel, quien piensa en un cuerpo policial basado en ideas de prevención en cooperación con la comunidad, (...) "La policía es la gente y la gente es la policía ${ }^{5}$ ". Esta filosofía distingue a la policía comunitaria, cuyo objetivo es superar el modelo de policía tradicional reactivo, introduciendo una idea de coproducir seguridad.

En el escenario actual, es el delito y sus implicancias el eje central de la preocupación de los gobiernos. En la agenda política el devenir de la delincuencia es casi como la metáfora bíblica, el alfa y omega, el principio y el fin. Entonces, es la policía como una herramienta del Estado moderno, la que asume dos funciones básicas y visibles: mantener el orden social mediante la aplicación de la ley y combatir la criminalidad $^{6}$. Esta segunda función se materializa en labores de prevención, represión, vigilancia y disuasión del crimen. Además de estas labores, se ha afirmado que la policía cumple una función social, como una de las más antiguas instituciones de protección social y expresión de autoridad, ligada a la sociedad que la ha creado. Serán las características sociopolíticas y culturales del medio en el que actúa las que han de definir sus objetivos, en forma de organización y en sus funciones. El valor de la intervención dependerá de la capacidad que muestre no tan solo en responder a las demandas de la ciudadanía, sino que también en la capacidad de adaptación de las transformaciones del entorno social ${ }^{7}$.

Estos rasgos llevan a la policía actual a buscar nuevas estrategias, para hacer más eficaz y eficiente su labor. Con el devenir del tiempo, el modelo policial sufrió numerosas crisis de legitimidad, las que han propiciado una serie de reformas, buscando la

4 Bayley, David, Padrões de Policiamento: uma análise comparativa internacional, Núcleo de Estudos da Violência - Universidade de São Paulo, 2001.

5 Muniz, Jaqueline, Ser Policial é, sobre todo una razâo de ser. Cultura e cotidiano da Policia Militar do Estado de Río de Janeiro. Tese de doutorado em ciências políticas, IUPERJ, Río de Janeiro, Brasil, 2001.

6 La Curbet, Jaume, La desmitificación de la Policia /en/ Seguretat sostenible, № 1. La sociedad, tanto como la propia policía, han venido alimentando durante muchos años el mito de que el control de la delincuencia era cuestión estrictamente policial y ahora, claro está, los ciudadanos responsabilizan a la policía del aumento incesante de los delitos, que mayor inseguridad les provocan".

7 Rico, José María, El rol policial. Ciudad y seguridad en América Latina, Flacso, Santiago, 2005. 
aprobación popular. Un buen ejemplo es el caso Estados Unidos, en donde el modelo comunitario ha pasado por altos y bajos $^{8}$. La policía ha tenido que luchar para ganar el apoyo de la ciudadanía, y relación entre esta y aquella, ha estado marcada por escándalos de corrupción. Como ha dicho Greene: "el problema policial no era tanto que la policía utilizara abusivamente la ley, sino que dejara de aplicarla de forma selectiva"."

El manejo político sobre la policía desencadenó en reformas administrativas para profesionalizar la institución y encuadrar su actuar al mandato legal. Esto produjo el distanciamiento de la clase política, y (como efecto no deseado) el alejamiento de la comunidad. Las reformas de este tipo hicieron que predominara un modelo reactivo (represivo) de vigilancia y control. Un modelo que llevó a la profesionalización de la institución, al uso de nuevas tecnologías y a la existencia de una carrera policial. Desafortunadamente, estos cambios tampoco dieron los resultados esperados. Persistió la distancia entre la policía y la comunidad y fue necesario admitir que el modelo de vigilancia no funcionaba; por cuanto no prevenía el delito e incluso generaba más inseguridad. Esta situación desencadenó que en las décadas de los 70 y 80 se desarrollaran acciones civiles de repudio en contra de la policía, y estas manifestaciones condujeron a que se comenzara a hablar nuevamente de la policía comunitaria ${ }^{10}$. En este momento resurgen los principios de Sir Robert Peel, como refundadores del actuar de la policía comunitaria. Para Chalom ${ }^{11}$, estos principios se traducen en: (i) la misión básica de la policía es prevenir la criminalidad y el desorden público; (ii) la capacidad de la policía de concretar la misión institucional depende de la aprobación pública de su actuar; (iii) la libre cooperación de la ciudadanía en el acatamiento de la ley para asegurar el respeto de la población; (iv) el apoyo público se logra no por complacencia, sino demostrando un absoluto e imparcial servicio a la ley; y, finalmente, (v) el uso de la fuerza policial se requiere solo y en grado necesario para cumplir con la ley, cuando la persuasión, exhortación y advertencias resultan ser insuficientes. Agrega Chalom que el modelo de policía comunitario y sus principios se representan en la figura del patrullero a pie, de quien se espera ha de asegurar el orden además de entrar en conexión directa con la comunidad ${ }^{12}$.

Una visión crítica sobre la policía comunitaria la encontramos en Aniyar de Castro $^{13}$, para quien este modelo es solo una solución oportunista en respuesta a los

\footnotetext{
8 Greene, Jack, La policía de proximidad en Estados Unidos: Cambios en la naturaleza, estructura y funciones de la policía, National institute of Justice, New York y Granada, 2006.

9 Greene, Jack, obr. cit.

10 Varela, Jorquera, Fernanda, Modelos internacionales de policía de orientación comunitaria, Subsecretaria de Carabineros, Santiago.

11 Chalom, Maurice, et al., Seguridad ciudadana, participación social y buen gobierno: El papel de la policía. Centro de las Naciones Unidas para los asentamientos humanos (HABITAT-UN), Centro Internacional para la prevención de la criminalidad, Ciudades más seguras, Ediciones Sur. Santiago. 2001.

12 Chalom, M, obr. cit.

13 Aniyar de Castro, Lolita, El abolicionismo en marcha: Nuevos modelos de control. Participación ciuda-
} 
constantes alzamientos de las minorías, las que protestaban por la constante discriminación policial. Otra visión, menos crítica, es la Brogden y Nijhar, para quienes la participación de la ciudadanía como apoyo a la labor policial resulta ser una opción conveniente si se quiere desarrollar estrategias de prevención del crimen de forma económicamente eficiente ${ }^{14}$.

La instauración del modelo policial comunitario también incidió en la promoción de los derechos humanos y ciudadanos. La participación comunitaria contribuyó a que el modelo se propagara a varios países de los distintos continentes y haber transformado al modelo en una verdadera filosofía de quehacer policial ${ }^{15}$. En este sentido, Trojanowicz señala que: "La Policía Comunitaria implica un nuevo contrato entre la policía y los ciudadanos (...) esta nueva filosofía descansa en la creencia de que la gente, en la comunidad, merece influir en el proceso policial, a cambio de su participación y ароуо ${ }^{16 "}$. Estas ideas son el corolario de un cambio en las estrategias policiales, es decir, políticas de actuación dirigidas a comunidades específicas, de implementación gradual. La participación de la comunidad no es para generar simpatías y estadísticas de aprobación, sino una forma de canalizar de manera eficiente la lucha contra la criminalidad. No se trata de que la comunidad tome decisiones policiales, sino que esta sea oída y se generen espacios de intercambio de ideas. Un cambio en la cultura policial, es, esto en la forma en que los policías entienden su quehacer. No solo en labores represivas, sino de índole preventiva.

\subsection{Características y objetivos del modelo}

Se ha sostenido que el eje del modelo es procurar el bienestar de la comunidad, elevando su calidad de vida. El accionar policial se dirige a la protección de la comunidad frente al crimen, considerando que la policía es parte de la comunidad. En tal sentido, Butler señala: "La misión de la policía comunitaria es aumentar la confianza y satisfacción de la policía entre sus habitantes ${ }^{17 "}$.

Autores como Bayley y Skolnick ${ }^{18}$, luego de examinar diversas experiencias en el orbe, plantean las siguientes características que debe presentar el sistema comunitario:

dana y policía comunitaria. El caso de América Latina /en/ Revista Société Internationale de Defense Sociale pour Politique Criminelle Humaniste, 2003.

14 Brogden, Mike; Nijhar, Preeti, Community Policing: National and International Models and Approaches, Willan Publishing, UK, 2005.

15 Neild, Rachel, Temas y Debates en la Reforma de la Seguridad Pública: Una guía para la sociedad civil, Washington Office on Latin America, Washington DC, 1998.

16 Trojanowicz, Robert, et al., Community Policing. A Comtemporary Perspective. Anderson Cincinnati Publishing Co. 2, 1998.

17 Butler, Tony, La Policía Comunitaria en el Reino Unido/en/ Gloucestershire, s.e., Febrero, 2000.

18 Bayley, David; Skolnick, Jerome, Theme and Variation in Community Policing /en/ Crime and Justice: a review of Research, vol 10, 1988. 
1. Prevención del crimen basado en la comunidad. La policía debe mostrar presencia en la ciudad, ser visible y accesible con el fin de reducir el miedo y disuadir el crimen. La comunidad debe ser capaz de reconocer a la policía, estar en contacto directo con ellos. En el caso de Chile a través del plan cuadrante se ha pretendido este acercamiento.

2. Reorientación del patrullaje. El patrullaje debe tener una conexión profunda con la comunidad, no solo responder a llamados de emergencia. La policía patrulla como una rutina instaurada, no solo ante situaciones de riesgo, sino para marcar presencia policial en las calles.

3. Aumentar la rendición de cuentas. El sistema no solo implica escuchar a la comunidad sino también aceptar críticas a su trabajo. Este punto es complejo de regular, por cuanto solo en la medida que el acercamiento policía-comunidad sea real, las personas sentirán confianza para plantear quejas. Para Bayley y Skolnick ${ }^{19}$ la apertura policial es un gran avance, por cuanto contrasta con la idea arraigada en los cuerpos policiales de que son ellos quienes mejor saben lo que se debe hacer para proteger a la comunidad.

4. Descentralizar el mando. La forma tradicional es descentralizar geográficamente (unidades, cuadrantes). Para que esta labor sea eficaz, se requiere que la policía tenga la libertad de actuar de acuerdo a su propia lectura de las condiciones locales. Esta idea exige que no se generen fórmulas centralizadas para afrontar los problemas, sino que las necesidades de la comunidad sean las que administren y orienten el actuar de los funcionarios. Actuar diversamente significa apartarse del modelo centralizado, es decir, utilizar la descentralización de los mandos como una oportunidad para mejorar la imagen pública del rol policial ${ }^{20}$.

La comunidad ha de sumir un rol activo dirigido por una parte al estudio de los problemas delictuales presentes en su entorno y además evaluar el desempeño de las actuaciones policiales. Esto puede ejecutarse a través de elementos operativos como: reuniones permanentes con las asociaciones comunitarias, capacitación para los vecinos en cuanto a prevención del delito, información sobre el patrullaje a pie, talleres de resolución alternativa de conflictos, instrumentos de recolección de datos, para conocer la opinión del vecindario y planificación de las necesidades y las acciones a implementar para cubrirlas ${ }^{21}$.

Para el buen funcionamiento del modelo comunitario, es necesario un actuar policial acorde. No se trata solo esperar la buena voluntad policial, sino que todos

\footnotetext{
19 Bayley, David, obr. cit.

20 Aniyar de Castro, Lolita, obr. cit.

21 Varela, Jorquera, Fernanda, obr. cit.
} 
los funcionarios deben actuar conforme a estas nuevas directrices. Los cambios se orientan a lograr un "estilo empresarial" en el actuar policial. En otras palabras, esto significa que la policía debe tomar riesgos, elevar sus estándares de respuesta y de satisfacción de los ciudadanos en sus actuaciones, descentralizar sus secuencias de mando y sus servicios ${ }^{22}$.

Greene $^{23}$ requiere además que la interacción de funciones entre la policía y la comunidad se fundamente en una relación de asociatividad (partnership), la que debe extenderse a otras agencias públicas y privadas que sirven e impactan en la comunidad y que se vinculan con la policía.

El proceso de apertura policial implica cambios en la gestión habitual de las cosas, por cuanto los nuevos desafíos y la participación de otros actores sociales, conllevan la necesidad de desarrollar nuevas dinámicas en los cuerpos policiales. En esta línea de pensamiento Greene ha dicho: "Estas nuevas habilidades se relacionan con un mayor poder de discrecionalidad de la policía, agregando que muchas veces se considera que el papel de la policía se ha incrementado desde ser solo un instrumento gubernamental a un instrumento de diagnóstico social y de movilización comunitaria ${ }^{24 \prime}$.

\subsection{Objeciones al modelo}

La instauración de un modelo de policía comunitaria genera resistencias ya que cambiar el paradigma de la activad policial, centrado en principio en acciones represivas a ideas preventivas, no es cuestión simple. Las principales dificultades son las siguientes ${ }^{25}$ :

1. La misma policía ve en el modelo comunitario solo un cambio de imagen y la asignación de tareas adicionales. Una posible solución puede ser la creación de incentivos, que los policía no vean esto como una recarga de trabajo ${ }^{26}$. La idea de combatir la cultura policial hace referencia al modo de entender el actuar policial; como se ha dicho, los policías piensan que solo ellos saben cómo aplicar la ley y qué es bueno para los ciudadanos.

2. En esta misma línea se presentan problemas en la estructura policial; así, los mandos medios, ven en el modelo una pérdida de su poder; la descentralización para ellos es el posicionamiento de los subalternos y pérdida en la seriedad de la

22 Bayley, David, obr. cit.

23 Greene, Jack, obr. cit.

24 Greene, Jack, obr. cit.

25 Varela Jorquera, Fernanda, obr. cit.

26 Skogan, Wesley, obr. cit. 
actividad policial, lo que ejemplifica el problema de la "cultura policial"; de tal forma, los policías en estas nuevas estrategias comunitarias, organizan charlas en los barrios acerca de lo problemas de las drogas, el alcohol, etc. Estas simples prácticas pueden resultar frustrantes para una policía acostumbrada a combatir, que ve estas actividades como un trabajo social27.

Relacionado con los problemas al interior de la policía, aparece otra dificultad. Los cambios de liderazgo generan conflictos por cuestiones administrativas internas (promociones y bajas) o bien cambios del gobierno central o local. Estos inconvenientes pueden poner en peligro los proyectos comunitarios ya instaurados y los avances obtenidos en la medida en que todo los cambios de administración implican una evaluación (y muchas veces la corrección) de la administración saliente.

3. Discusión provoca la evaluación de las prácticas comunitarias tanto en su dimensión real como en los resultados generados. ¿Cómo se mide el impacto de sus estrategias? ¿Su efectividad en el medio en el que son incorporadas? Aunque existen ciertos progresos al respecto, en los últimos años "gracias a las investigaciones vinculadas a los estudios de victimización, del temor al delito, a la satisfacción del público o de los policías" 28 es evidente la falta de herramientas que permitan medir o cuantificar resultados.

4. Otro asunto problemático para el modelo, es la escasa cooperación de los municipios con los diversos servicios policiales, ya sea por razones burocráticas, políticas, presupuestarias. Skogan ${ }^{29}$ señala que este es uno de los problemas más difíciles de superar para los promotores del policiamiento comunitario, pues si la implementación solo dependiera de los esfuerzos policiales, este sería un fracaso. En este entendido, la participación de la comunidad no es automática, pues debemos considerar las resistencias históricas, culturales, entre la policía y comunidad. Sí se quiere una relación de poder y jerarquía, donde los ciudadanos han sido los súbditos. Se hace necesario, entonces, promocionar y difundir las nuevas estrategias para producir un acercamiento gradual de la comunidad. Aquí la publicidad juega un rol activo en lograr persuadir a los ciudadanos de los beneficios que trae la implementación del modelo.

27 Normandeau, André, La Policía Comunitaria en Norteamérica /en/ Cuadernos de Criminología, Policía de Investigaciones de Chile, Instituto de Criminología, No 5, Chile, 1995.

28 Normandeau, André, obr. cit.

29 Skogan, Wesley, obr. cit. 


\section{El caso chileno}

Estas ideas han tomado fuerza en Carabineros de Chile. Sin embargo, el plan cuadrante no implementa un modelo de policía comunitaria puro. En esta institución se sigue lo que se ha denominado "community policing", que es una variante moderada del modelo, que consiste en involucrar a la sociedad en una labor más estrecha con la policía sin hacer reformas profundas en el funcionamiento institucional ${ }^{30}$.

Carabineros de Chile es una institución policial centralizada, uniformada y castrense, compuesta por alrededor de unos 37.000 mil funcionarios, enmarcada dentro de una estructura político-administrativa también centralizada ${ }^{31}$.

El momento cúlmine para la asunción de los postulados del modelo comunitario en esta policía, se dio en el año 2001 (el inicio de estos planes se remontan a la década de los 90 con la implementación del Plan cuadrante de seguridad preventiva ${ }^{32}$ (plan enmarcado dentro de un plan estratégico nacional ${ }^{33}$ ). Los objetivos específicos del plan son dos: potenciar la vigilancia policial preventiva, es decir, aumentar los controles informales, involucrando a los miembros de la comunidad en la solución de las problemáticas delictuales mediante la responsabilización de sus miembros, y dinamizar el actuar policial para dar respuesta oportuna y eficaz a los requerimientos de la comunidad, lo cual evita una sobrerreacción policial.

Se puede señalar que el diseño del Plan cuadrante se sitúa dentro de los principios del modelo de policía comunitaria. Se reconocen en este plan estratégico las siguientes similitudes: la descentralización del mando, la intervención comunitaria enfocada en la presencia policial visible en la comunidad; la interacción entre la policía y la comunidad a través de la generación de espacios de cercanía y comunicación. Revisaremos cada una de ellas en las siguientes líneas:

1. Para la implantación del Plan cuadrante $^{34}$ se hizo necesaria la división del territorio jurisdiccional de cada comisaría; estas se subdividieron en sectores Ilamados cuadrantes que corresponde al territorio entregado a la vigilancia de

30 "Si se quiere que las fuerzas policiales participen eficazmente en la construcción de aglomeraciones urbanas seguras y sustentables, habrá que tomar en consideración una serie de aspectos que constituyen los desafíos de un buen gobernar. Ellos se relacionan con una transformación cualitativa de las organizaciones policiales, el mejoramiento de sus relaciones con la sociedad civil y la participación de la policía como actor cooperador esencial en el desarrollo urbano". Chalom, Maurice, et. al., obr. cit.

31 Ver www.carabineros de chile.cl

32 Días, Javiera, Relación Policía-comunidad. Análisis de la experiencia del plan cuadrante en Chile [on line]. Disponible en la World Wide Web: http://www.policiasysociedad.org/userfiles/javieradiaz_esp.pdf

33 Ver www.plan cuadreante.cl

34 Varela, Jorquera, Fernanda, obr. cit. 
una dotación específica de policías. La división precedente no es arbitraria, sino que está fijada en razón de características geográficas y demográficas (territorio, número de personas residentes), para luego integrar información respecto a ocupación de la población residente, edad, actividades económicas, detectar sitios de conflicto o peligro (espacios abandonados, tipologías delictivas presentes en la zona). Permite así que la policía intervenga en los espacios materiales, ya que conociendo el perímetro se inhibirán posibles delitos que se ven favorecidos por algunas condiciones materiales ${ }^{35}$.

El conocimiento de las personas que integran el grupo es fundamental, ya que así se podrá actuar de acuerdo a las necesidades del grupo comunitario ${ }^{36}$. Se produce un manejo eficiente de los recursos y estrategias. No es igual intervenir en una ciudad como Santiago, que en una comunidad como, por ejemplo, Isla de Pascua. Acorde con esto, la asimilación debería ser gradual, porque la comunidad ha de sentirse paulatinamente involucrada ${ }^{37}$.

2. La asignación de recursos materiales tiene relación con las características de los cuadrantes, los cuales determinan el tipo de vigilancia: motorizado, en vehículo o a pie. La distribución de los recursos humanos o materiales es de acuerdo a los requerimientos de cada cuadrante. Estos se clasifican en base a niveles específicos de intervención en razón de delincuencia presente en el cuadrante. De acuerdo al nivel en que se clasifica el cuadrante, se le asignan las Ilamadas Unidades de Vigilancia Equivalentes (U.V.E.), las cuales expresan un valor que determina el tipo de recurso, es decir, mientras mayor sea el nivel de frecuencia de delitos en el cuadrante, mayor cantidad de U.V.E. se le asignarán. Este sistema implica una interacción policía-comunidad acorde con las necesidades puntuales del sector, así como una presencia policial visible. La policía como socio comprometido de la comunidad ${ }^{38}$.

Es común a todos los cuadrantes la duración del servicio, el cual consiste en tres turnos de 8 horas, de las cuales 6 horas corresponden a movimiento y desplazamiento por la zona y 2 horas en detención. Sin embargo, la frecuencia de las rondas también está determinada por el nivel al cual pertenece el cuadrante.

3. Mejorar la gestión preventiva de cada cuadrante, significa que el personal a cargo de este obtenga una progresiva identificación con los vecinos y la zona a su cargo. Este conocimiento permite obtener información relevante captada del contacto directos con los vecinos y lograr que la comunidad reconozca a sus

35 Laurrari, Elena; Cid, José, Teorías Criminológicas, Ed. Bosch, Barcelona, España, 2001.

36 Muniz, Jaqueline, obr. cit.

37 Varela, Jorquera, Fernanda, obr. cit.

38 Ídem. 
policías, sintiéndolos comprometidos, accesibles, francos y profesionales en el ejercicio de las funciones encomendadas ${ }^{39}$.

\section{Conclusiones}

La implementación de un modelo de policía comunitaria no es tarea fácil, porque lo que funciona para determinados sistemas policiales, no es garantía que para otros sea igualmente eficaz. La adopción de una nueva estrategia en materia de acción policial requiere ciertos ajustes. Ajustes que provienen desde el examen de los mismos principios que sustentan el modelo.

Una reforma a la institución policial en sede comunitaria, es una cuestión paulatina, no es un milagro, por cuanto no se trata de estrategias o una lista de actividades a seguir, sino más bien de una nueva filosofía policial. Filosofía que implica cambios profundos en los agentes involucrados. Cambios que en lo que respecta a la institución policial y sus miembros es transversal, entendiendo que las instituciones policiales tienen sus propios parámetros culturales y que el acercamiento a la comunidad no es fácil. Esta filosofía o cultura policial no es otra cosa que cómo entiende la policía su actividad, cómo ven ellos su misión institucional. Desmitificar ideas tales como que son ellos los únicos que saben qué es los mejor para la ciudadanía, puesto que ellos conocen la ley.

Faltan estudios empíricos que indiquen la efectividad de estos planes en lo que es la preocupación fundamental de las sociedades actuales, la prevención y reducción de la criminalidad. En sociedades como la latinoamericana, es un avance que la policía gane la confianza de la comunidad, que a través de este contacto directo pueden idearse fórmulas eficaces para problemas cotidianos, considerando las especiales condiciones de cada grupo.

La descentralización de la policía y sus mandos es útil como medio de manejar mejor los recursos. Además de permitir adecuar, por una parte, la intervención y, de otra, las soluciones en relación a las necesidades concretas. Estos planes han de tener cierta continuidad y no estar sujetos al gobierno de turno, no transformarse en acciones que solo buscan la legítima temporal, la simpatía del electorado.

Para el caso de Chile, se observa una reestructuración policial, que ha venido avanzando en el tiempo. Siguiendo a Patricio Tudela ${ }^{40}$, la actuación policial se ha transformado en un tema nacional. Mediante la integración y participación real de la

39 Ídem.

40 Tudela, Patricio, Sociedad y Policía: Desarrollo y retos de la función policial en las democracias latinoamericanas, 2007. 
sociedad civil en las policías se ha iniciado un proceso de acercamiento a los contenidos de "community policing". Como se ha señalado, la supervisión ciudadana sobre la policía es un factor clave para el policiamiento comunitario; en consecuencia, la rendición de cuentas públicas orientadas a la responsabilización, la identificación de problemas y su solución, son procesos que deben estimularse.

Carabineros de Chile se ha involucrado en un proceso de policiamiento comunitario reflejado en la aplicación del Plan cuadrante ${ }^{41}$. Las tareas implementadas responden a una nueva manera de abordar el fenómeno delictual, cuestión que involucra a todos los miembros de la comunidad. En general, la cooperación entre los actores en sus nuevos roles es fundamental, ya que el objeto de todas estas prácticas no es otro que procurar el bienestar comunitario lo que a fin de cuentas significa que otras motivaciones deben abandonarse.

\section{Bibliografía}

Aniyar de Castro, Lolita, El abolicionismo en marcha: nuevos modelos de control. Participación ciudadana y policía comunitaria. El caso de América Latina /en/ Revista Sociètè Internationale de Defense Sociale pour une Politique Criminelle Humaniste, 2003.

Bayley, David, Padrões de Policiamento: uma análise comparativa internacional, Núcleo de Estudos da Violência - Universidade de São Paulo, 2001.

Butler, Tony. "La Policía Comunitaria en el Reino Unido". Gloucestershire: s.e., febrero, 2000.

Chalom, Maurice, et al., Seguridad ciudadana, participación social y buen gobierno: El papel de la policía. Centro de las Naciones Unidas para los asentamientos humanos (HABITAT-UN), Centro Internacional para la prevención de la criminalidad, Ciudades más seguras, Ediciones Sur. Santiago. 2001.

FrüHling, Hugo, Dos Décadas de Reforma Policial en América Latina: Factores para su éxito o fracaso, Banco Interamericano del Desarrollo BID. Washington, 2007.

Frühling, Hugo, Policía Comunitaria y Reforma Policial en América Latina. ¿Cuál es el impacto? Centro de estudios de seguridad ciudadana. Universidad de Chile, Santiago, Chile, 2003.

Greene, Jack, La Policía de Proximidad en Estados Unidos: Cambios en la naturaleza, estructura y funciones de la policía, National Institute of Justice. New York y Granada, 2006.

41 Según informe del primer semestre del año 2009, emitido por Carabineros de Chile, 88 comunas de todo el país cuentan con el Plan cuadrante. Véase www.plancuadrante.cl 
EL MODELO DE POLICÍA COMUNITARIA: EL CASO CHILENO

Muniz, JaCqueline, Ser Policial é, sobre todo uma Razão de Ser, Cultura e cotidiano da Polícia Militar do Estado de Rio de Janeiro, Tese de Doutorado em Ciências Políticas, IUPERJ, Rio de Janeiro, 2001.

Neild, Rachel, Temas y Debates en la Reforma de la Seguridad Pública: Una guía para la sociedad civil, Washington Office on Latin America, Washington DC, 1998.

Normandeau, André, La Policía Comunitaria en Norteamérica /en/ Cuadernos de Criminología, Policía de Investigaciones de Chile Instituto de Criminología, № 5. 1995.

Rıco, José María, El Rol Policial /en/ Ciudad y Seguridad en América Latina, FlacsoChile, 2005.

Skogan, Westey, Réformer la Police et la sécurité. Les nouvelles tendences en Europe et États-Unis. Odile Jacob, 2004.

Skolnick, Jerome; Bayley, David, Theme and variation in Community Policing /en/ Crime and Justice: a review of Research, vol. 10, 1988.

Tudela, Patricio, Sociedad y Policía: Desarrollo y retos de la función policial en las democracias latinoamericanas, 2007

Trojanowicz, Robert, et al., Community Policing. A Comtemporary Perspective. Anderson Cincinnati Publishing Co., 2, 1998.

Varela, Jorquera, Fernanda. Modelos internacionales de policía de orientación comunitaria. Subsecretaría de Carabineros. 